\title{
DIGITAL VIDEO WATERMARKING USING MODIFIED LSB AND DCT TECHNIQUE
}

\author{
Priya Porwal ${ }^{1}$, Tanvi Ghag ${ }^{2}$, Nikita Poddar $^{3}$, Ankita Tawde $^{4}$ \\ ${ }^{1}$ Assistant Professor (Comp. Dept) MPSTME, Mumbai, India \\ ${ }^{2}$ Student, 4th Year B.Tech Computer Engineering, MPSTME, Mumbai, India \\ ${ }^{3}$ Student, 4th Year B.Tech Computer Engineering, MPSTME, Mumbai, India \\ ${ }^{4}$ Student, 4th Year B.Tech Computer Engineering, MPSTME, Mumbai, India
}

\begin{abstract}
From the previous millennium there has been a significant change from the analog to digital world. In today's time Audio $c d$ 's, Internet and DVD's are more widespread than before. Though the film and music content owners are reluctant to release digital contend. This situation is because if this digital content is left unprotected, there are high chances of it being copied rapidly, on a large scale, with no limitations on the number of copies and it can be easily distributed via Internet. [3]As there is a rapid development in the current Information Technology, electronic publishing like the distribution of digitized images and videos, are becoming more and more popular. So there is a need of protecting as well as authenticating 3 dimensional data. Copyright protection is an important issue for electronic publishing.

This paper presents the different video watermarking techniques. It provides a review on various available algorithms. In addition to it, focus on Combined Least Significant Bit (LSB) and Discrete Cosine Transform(DCT) Technique and also LSB with modifications Technique.
\end{abstract}

Keywords: Significant Bit (LSB), Discrete Cosine Transform (DCT), Watermarking, Video, Copyright Protection

\section{INTRODUCTION}

One of the current copyright protection methods that has recently received considerable attention is Watermarking. The multimedia can be anything for example, it can be text, audio, video and their compositions as well. Conventional watermarks are generally found on valuable paper documents like our check, stock certificates and cash.[1] These conventional watermarks can be viewed by a certain angle or through certain illumination. They cannot be easily removed without leaving an evidence of being tampered. Some watermarks can be visible to indicate ownership of Digital images/video using translucent logos generally used by some television broadcast station superimposed on their transmitted videos. These are plain in view and they do not provide any form of equivalent physical properties other than the actual pixel values to modify. In this case if any pixel values are changed, the content changes as well.[8] A balanced should be maintained so that the watermark imposed can be clearly visible but at the same time difficult to remove. Another concern for visible watermark is that it shouldn't cause any kind of visual distractions. Invisible watermarks are different from visible watermarks, although their objectives are the same to provide copyright protection. Visible watermarks can be used to help prevent any form of piracy by showing ownership semi-transparently on top of images
Various areas where watermarking can be applied:

- Audio

- Video

- Documents

- Images

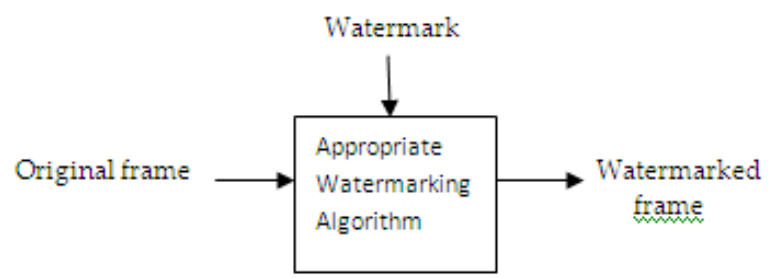

Fig- 1: Basic watermarking block diagram

\section{TYPES OF WATERMARKING}

Types of watermarking

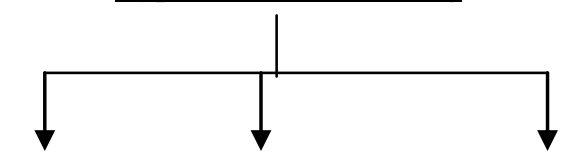

Visible Invisible Visible Partially 


\subsection{Visible Watermarking}

Watermarks are applied to images only. These watermarks can be seen and they cannot be removed by just cutting out the watermark. They are protected from statistical analysis.[5] The only disadvantage of visible watermark is it degrades the image quality. It is not possible to detect them by dedicated programs or devices.

\subsection{Invisible Watermarking}

These watermarks are not visible and they can be removed only by the authorized user. They are used by authors and content protectors as they help in protecting the content from being copied.

\subsection{Partially Visible Watermark}

The partially visible watermark is a combination of a visible watermark and an invisible watermark. First a visible watermark is inserted in the host image and then an invisible watermark is added to the already visible-watermarked image.

\section{WATERMARKING DOMAINS}

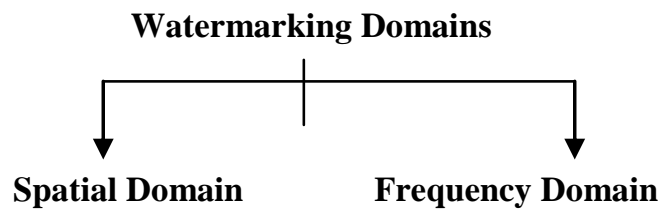

\subsection{Spatial Domain}

In this technique the watermark embedding is achieved by modifying directly pixel values of host image. Most commonly used method in this technique is the least significant bit (LSB) method.[9] In this technique the least significant bit (LSB) of each pixel in host image is modified so as to embed the secret message. This can help resist only some attacks. In this, the watermark is embedded into the original image in spatial domain, dividing the original image into different sizes of block and by adjusting the brightness of the block according to the watermark embedded.[4] The methods proposed are robust enough against some operations of image processing, like median filter, scaling and rotation; they are however less robust to cropping attack as the bits of watermark are embedded in the whole image therefore some data may be lost in cropping.

\subsection{Frequency Domain}

In this technique, host image is initially converted into frequency domain by using the method of transformation like the discrete wavelet transforms (DWT),discrete cosine transforms (DCT) or discrete Fourier transforms (DFT) . [7] The transform domain coefficients are then modified using the watermark. The inverse transform is applied to obtain the image watermarked finally. Due to complicated calculations done by the forward and inverse transforms, these methods are more complex and have higher computational costs compared to spatial domain methods. Transformation domain methods however are more robust enough against attacks compared to spatial domain methods.

Commonly used Transform domain methods:

-DCT - Discrete Cosine Transform

-DWT - Discrete Wavelet Transform

-DFT- Discrete Fourier Transform

\section{COMBINED MODIFIED LSB AND DCT WATERMARKING TECHNIQUE.}

General LSB technique is used because it is used from so many years $\&$ it is well trusted. This technique gives the ease of use \& simplicity of coding with precise results.[12] This technique provides the result in both visible \& invisible form (As per the requirement). With some modifications it is good to incorporate this technique for video watermarking.

How the water mark is inserted using LSB technique.

- First the video is divided in number of frames using MATLAB function in bmp file format(bmp file format is uncompressed file format \& this format restores the binary data in much safe way).

- Then the number which we need to encrypt (as a key) is converted in binary form.

- This key number is then placed in message at LSB position.

- Then for each frame, message is watermarked at respective LSB position.

- At end all frames are again combined and video is formed with uncompressed file format to restore the data integrity.

- While extracting we get the exact message image and the key encrypted into the message image.

\section{Limitations:}

- Only $256 \times 256$ size video with such size image is good.

- Gray colour image \& video are better.

In Discrete cosine transform there is a conversion of a sequence of data points in the spatial domain to a sum of sine and cosine transforms with different amplitudes in the frequency domain[10].

\section{Algorithm for Watermark Embedding:}

The steps used in this technique are following:

- Read the colour host frame i.e. cover frame, Select a grayscale bitmap image of size $256 * 256$.

- Get the RGB components. 
- Read the watermark image, Select another grayscale bitmap image of size $64 * 64$.

- Apply level 2 DCT transform to cover object image to decompose the image d into blocks of $4 * 4$.

- Embedding the watermark components into the frequency sub components.

- Apply level 2 IDCT.

- Get the watermarked image.

- Calculation of MSE.

\section{DCT Encoding}

The general equation for level 1 DCT is defined as follows:

$$
F(u)=\left(\frac{2}{N}\right)^{\frac{1}{2}} \sum_{i=0}^{N-1} \Lambda(i) \cdot \cos \left[\frac{\pi \cdot u}{2 \cdot N}(2 i+1)\right] f(i)
$$

Fig- 2. Level 1 DCT

The general equation level 1-IDCT transform is simple $F^{-1}(u)$, is as follows

$$
\Lambda(i)= \begin{cases}\frac{1}{\sqrt{2}} & \text { for } \xi=0 \\ 1 & \text { otherwise }\end{cases}
$$

Fig -3. Level 1-IDCT

The general equation for a level 2DCT is defined as follows:

$F(u, v)=\left(\frac{2}{N}\right)^{\frac{1}{2}}\left(\frac{2}{M}\right)^{\frac{1}{2}} \sum_{i=0}^{N-1} \sum_{j=0}^{M-1} \Lambda(i) \cdot \Lambda(j) \cdot \cos \left[\frac{\pi \cdot u}{2 \cdot N}(2 i+1)\right] \cos \left[\frac{\pi \cdot v}{2 \cdot M}(2 j+1)\right] \cdot f(i, j)$

Fig -4. Level 2 DCT

The general equation level 2-IDCT transform is simple $F$ ${ }^{I}(u, v)$, as follows

$$
\Lambda(\xi)= \begin{cases}\frac{1}{\sqrt{2}} & \text { for } \xi=0 \\ 1 & \text { otherwise }\end{cases}
$$

Fig -5. Level 2 IDCT

The operations in the DCT are as follows:

- The host image is an $\mathrm{N}^{*} \mathrm{M}$ image;

- $f(i, j)$ represents the intensity value of the pixel in a row by column i.e. (i,j) form;

- Function $F(u, v)$ represents the DCT coefficient in a row by column i.e. $(\mathrm{k} 1, \mathrm{k} 2)$ of the following DCT matrix.

- For most of the images, much of the signal energy lies majorly at low frequencies, since they appear in the upper left corner of the DCT.
- The lower right values represent higher frequencies hence compression is achieved at this range, and are often small in size. So small that it is enough to be neglected with little visible distortion.

- The DCT input is an $8 * 8$ array of integer .8 bit pixels have levels from 0 to 255 since we are presenting the gray scale level. Therefore an 8 point DCT would be:

$$
\Lambda(\xi)= \begin{cases}\frac{1}{\sqrt{2}} & \text { for } \xi=0 \\ 1 & \text { otherwise }\end{cases}
$$

Fig- 6. 8 point DCT.

DCT based watermarking techniques are of two types Global DCT watermarking and Block based DCT watermarking. The technique implemented in our project is Block based DCT.[11] The major merit of DCT technique is its robustness against simple image processing changes like brightness, contrast adjustment, blurring etc. The major flaw with this technique is its low resistance against physical changes that could be cropping or scaling etc.

In this technique we use a combination of both LSB and DCT Techniques.

Proposed system steps:

1. Divide video into frames.

2. Select the watermark image

3. Watermark each frame using LSB Technique first

4. Simultaneously watermark each frame using DCT Technique.

5. Combine the frames back into video

6. Extract watermarks

i. Extracted watermark from lsb technique

ii. Extracted watermark from dct technique. 


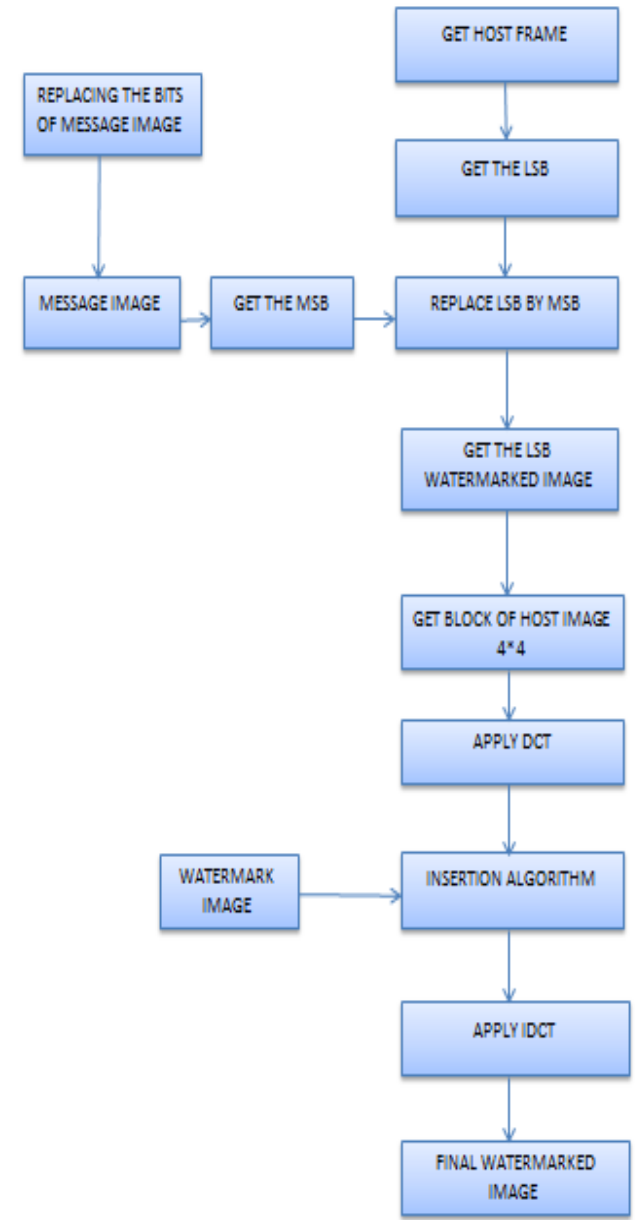

Fig- 7 Flowchart for LSB and DCT Technique

\section{RESULTS AND STUDIES.}

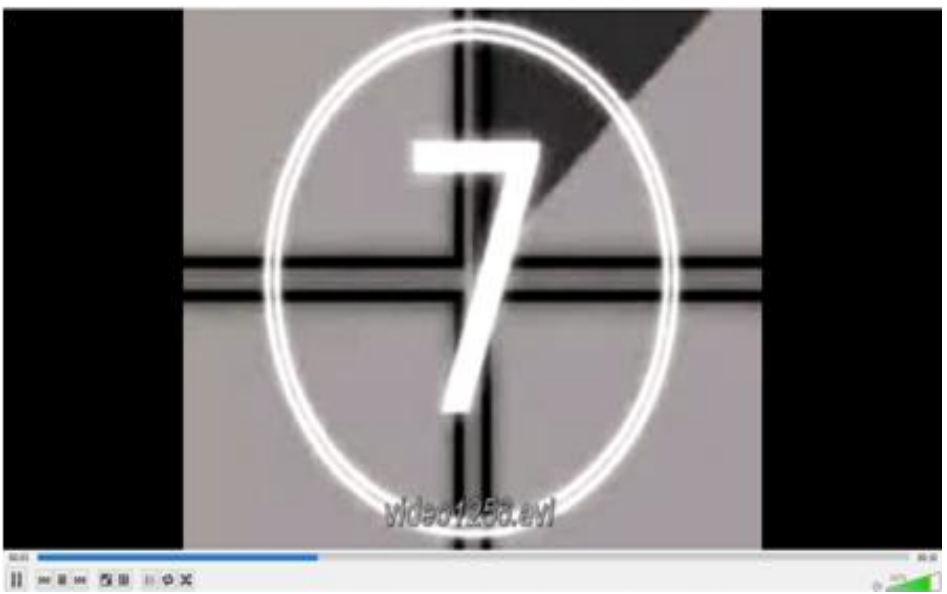

Fig- 8 Original Video

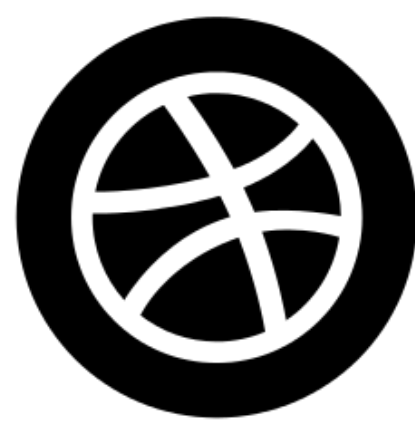

Fig- 9 Original Message Image

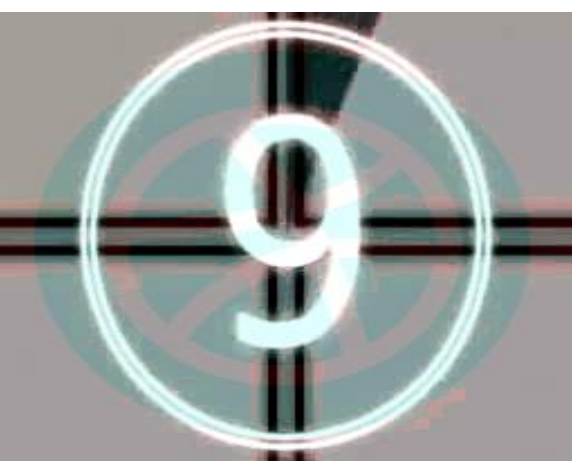

Fig- 10 Watermarked Video

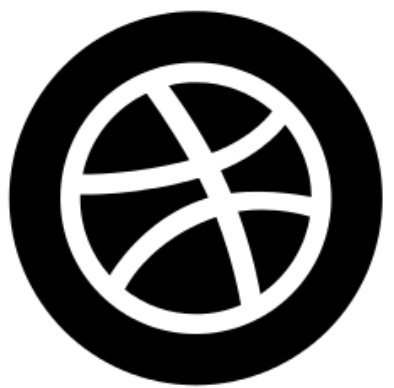

Fig -11 Extracted watermark using LSB

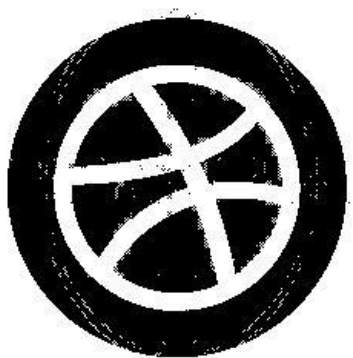

Fig 12 Extracted watermark using DCT

Mean Squared Error of the video with this technique is 13.6566. 


\section{CONCLUSIONS}

Watermarking is an active research field which has a lot of applications. Even though it is considered as a relatively new field, it still has produced some very important algorithms for hiding messages in digital signals. They can be described using many different models. [13]Four of the broad categories for these models were described in this black book. We have implemented four techniques using MATLAB on the video. We have proposed the Least Significant Bit Technique, Discrete Cosine Transform Technique, Combined Least Significant Bit and Discrete Cosine Transform Technique and Least Significant Bit with Modifications. Out of all the above four proposed techniques Discrete Cosine Transform watermarking techniques in frequency domain have better perceptual quality control and capacity than the others. Hence, this technique is a better option compared to the others.

Digital watermarking has key importance in protecting digital content. In a watermarking system tampering a watermark should be detectable always, and trying to remove a watermark from the host frame should cause host frame to be useless .Watermarking research is progressing exponentially and many researchers and developers are focusing in developing schemes to provide the owners of digital content guarantee of copyright.

\section{REFERENCES}

[1]. Abdullah Bamatraf, Rosziati Ibrahim and Mohd. NajibMohd. Salleh, (2011) 'A New Digital Watermarking Algorithm Using Combination of Least Significant Bit (LSB) and Inverse Bit' ,JOURNAL OF COMPUTING, VOLUME 3, ISSUE 4, APRIL 2011, ISSN 2151-9617.

[2]. Chirag Sharma and Deepak Prashar,(2012) 'Efficient Watermarking Technique for Digital Media (Images and Videos)' ,International Journal on Computer Science and Engineering (IJCSE), Vol. 4 No. 05.

[3]. Cox.I.J, Linnartz.J.G, (1998), "Some general methods for tampering with watermarks", IEEE Journal on Selected Areas in Communications, 16(4), pp. 587-593.

[4]. Feng Liu,Yongtao Qian, (2011) “A Novel Robust Watermarking Algorithm Based On Two Levels DCT and Two Levels SVD", Third International Conference on Measuring Technology and Mechatronics Automation .

[5]. Gurpreet Kaur and Kamaljeet Kaur,(2013) , 'Image Watermarking Using LSB (Least Significant Bit)',International Journal of Advanced Research in Computer Science and Software Engineering, Volume 3, Issue 4 pp. 858861.

[6]. Hamid Shojanazeri, Wan Azizun Wan Adnan, SharifahMumtadzah Syed Ahmad, (2013) 'Video Watermarking Techniques for Copyright protection and Content Authentication', International Journal of Computer Information Systems and Industrial Management Applications.,ISSN 2150-7988 Volume 5 (2013) pp. 652-660.
[7]. Jayamalar T, Radha V (2010) "Survey on Digital Video Watermarking Techniques and attacks on Watermarks", International Journal of Engineering and Technology, Vol. 2(12), Pp 6963-6967,2010.

[8]. Jiang, A Armstrong, (2002), "Data hiding approachfor efficient image indexing”, Electronics letters. 7th,38(23), pp. 1424-1425.

[9]. Karnpriya Vyas ,Kirti Sethiya and Sonu Jain, (2012), 'Implementation of Digital Watermarking Using MATLAB Software' , AN INTERNATIONAL JOURNAL OF ADVANCED COMPUTER TECHNOLOGY, 1 (1), VolumeI, Issue-I (2012).

[10]. Kesavan Gopal, Dr. M. Madhavi Latha(2010) "Watermarking of Digital Video Stream for Source Authentication" IJCSI International Journal of Computer Science Issues, Vol. 7,Issue 4, No 1.

[11]. Keshav S Rawat, Dheerendra S Tomar, "Digital watermarking schemes for authorization against copying or piracy of color images" Indian Journal of Computer Science and Engineering Vol. 1 No. 4 , pp-295-300 .

[12]. Prachi V. Powar and Prof.S.S.Agrawal, (2013), 'DESIGN OF DIGITAL VIDEO WATERMARKING SCHEME USING MATLAB SIMULINK ' ,International Journal of Research in Engineering and Technology ,Volume: 2 Issue: 5, ISSN: 2319 - 1163 , pp. 826 - 830.

[13]. Sathik M. Mohammed and Sujatha S.S,(2010)"An Improved Invisible Watermarking Technique for Image Authentication", International Journal of Advanced Science and Technology,Vol-24,Pp-61-74,2010.

[14]. W. Bender, D. Gruhl, and N. Morimoto,( 1994) “Techniques for data hiding," Tech Rep., MIT Media Lab. 